\title{
Pimenteira ornamental submetida a tratamentos com daminozide em vasos com fibra de côco ou areia
}

\section{Ornamental pepper submitted to treatments with daminozide in pots containing coconut fiber or sand}

\author{
Ingrid Bernardo de Lima ${ }^{1 *}$; Arivaldo Braga dos Santos ${ }^{2}$; Julianny Jéssyca \\ Sousa da Fonseca ${ }^{2}$; Roberto Jun Takane ${ }^{3}$; Claudivan Feitosa de Lacerda ${ }^{4}$
}

\section{Resumo}

Cresce a procura por pimenteiras ornamentais no mercado de plantas envasadas e compactas, sendo possível sua obtenção através do uso de reguladores de crescimento associados ao cultivo em substratos de fácil aquisição e capazes de proporcionar mudas de alta qualidade a baixo custo. Objetivou-se avaliar o efeito das aplicações e concentrações do regulador de crescimento daminozide em plantas de pimenta ornamental cultivadas em vasos contendo como substratos fibra de côco (FC) ou areia. Utilizou-se o delineamento inteiramente casualizado arranjados em esquema fatorial $2 \times 5$, constituído por dois tipos de substrato (FC e areia) e cinco concentrações de daminozide $\left(0 ; 2 ; 4 ; 6\right.$ e $\left.8 \mathrm{~g} \mathrm{~L}^{-1}\right)$, aplicadas três vezes ao longo do ciclo, aos 25,40 e 55 dias após a semeadura. Foram avaliadas as seguintes variáveis: altura e diâmetro do caule; número, largura e comprimento dos frutos; massa seca dos frutos, folhas, caules e raízes, índice relativo de clorofila, fotossíntese liquida, condutância estomática, concentração interna de $\mathrm{CO}_{2}$, taxa de transpiração e temperatura das folhas. Daminozide reduziu a altura das plantas de pimenta em ambos os substratos, sendo a fibra de côco o substrato que proporcionou plantas de maior vigor e desenvolvimento. Com o aumento das concentrações do regulador, plantas de pimenta apresentaram caules mais espessos, menor altura e área foliar, folhas com maiores índices de clorofila, redução das seguintes variáveis: largura e comprimento dos frutos, taxa de transpiração, fotossíntese líquida e condutividade estomática das plantas.

Palavras-chave: Floricultura, Capsicum annuum, inibidor de giberelina, regulador de crescimento, substrato

\begin{abstract}
The demand for ornamental pepper as "potted plants" is growing. It is possible to produce these plants with growth regulators in substrates easily acquired and capable of providing high quality plants at low cost. The present study had the goal of evaluating the effect of applications of levels of the regulator daminozide on ornamental pepper grown in pots containing coconut fiber (FC) or sand as substrates. A completely randomized design was employed in a $2 \times 5$ factorial scheme consisting of two types of substrate (FC and sand) and five levels of daminozide $\left(0,2,4,6\right.$ and $\left.8 \mathrm{~g} \mathrm{~L}^{-1}\right)$ applied three times during the experiment, at 25,40 , and 55 days after sowing. The following variables were analyzed: plant height, stem diameter, number, width and length of fruits, dry matter of leaf, stem, fruits, and root,
\end{abstract}

\footnotetext{
${ }^{1}$ Eng $^{\mathrm{a}}$ Agr ${ }^{\mathrm{a}}$, Discente de Doutorado no Curso de Pós Graduação em Agronomia, Fitotecnia, Universidade Federal do Ceará, UFC, Fortaleza, CE. E-mail: ibl ingrid@hotmail.com

${ }^{2}$ Discentes do Curso de Graduação em Engenharia Agronômica, UFC, Fortaleza, CE. E-mail: arivaldobraga@gmail.com; julianny_sousa@hotmail.com

${ }_{3}^{3}$ Prof. Dr. do Dept ${ }^{\circ}$ de Fitotecnia, UFC, Fortaleza, CE. E-mail: robertotakane@ufc.br

${ }^{4}$ Prof. Dr. do Dept ${ }^{\circ}$ de Engenharia Agrícola, UFC, Fortaleza, CE. E-mail: cfeitosa@ufc.br

*Autor para correspondência
} 
leaf area, relative chlorophyll index, net photosynthesis, reduced stomatal conductance, internal CO2 concentration, transpiration rate and temperature of leaves. Daminozide reduced plant height pepper on both substrates, and the coconut fiber provided greater vigor and development. With increasing concentrations of the regulator pepper plants had more thicker stems, lower height and leaf area, leaves with higher chlorophyll content, and presented reduction in the following variables: width and length of fruits, rates of transpiration, net photosynthesis, and stomatal conductance of plants.

Key words: Flower farming, Capsicum annuum, inhibitor of gibberellins, growth regulator, substrates

\section{Introdução}

A princípio, todas as pimentas (Capsicum annuum) podem ser utilizadas como ornamentais $\mathrm{e}$ tem ocorrido um significativo aumento na procura e na aceitação dos consumidores quanto ao seu uso como planta ornamental de vaso (UPNMOOR, 2003), sendo as de porte baixo, com frutos coloridos, eretos e vistosos as mais procuradas e apropriadas para este mercado (VIEIRA, 2002). Dentre os méritos das pimenteiras como ornamentais de vasos destacam-se a fácil propagação por sementes, o curto período de cultivo, a tolerância ao calor e a seca, mantendo excelente qualidade, harmonia de vaso e fácil manutenção (GROSSI et al., 2005; STOMMEL, BOSLAND, 2006).

Quando plantas em vasos apresentam altura maior que o desejado, o uso de reguladores do tipo redutores de crescimento faz-se necessário (TOLOTTI; BELLÉ; MAINARDI, 2003), tornando-se uma prática comum na busca por plantas compactas e atraentes (GROSSI et al., 2005) e eficaz por reduzir o crescimento longitudinal de internódios, modificando aspectos funcionais e qualitativos de várias espécies (OZGUR, 2011), como por exemplo a abertura e fechamento de estômatos e número de frutos ou inflorescências. Assim, estes produtos são capazes de modificar a dinâmica interna do crescimento e desenvolvimento das plantas (DAVIS; STEFFENS; ANKHLA, 1988; DAVIS; CURRY, 1991), sem caráter fitotóxico, causando reduções nos níveis endógenos de giberelinas, culminando em reduções em altura, no alongamento e divisão celular das hastes, pecíolos e pedúnculos, e alterações na morfologia internas e anatômicas dos vegetais (RADEMACHER, 2000).
$\mathrm{O}$ produto daminozide, conhecido comercialmente como B-nine, Alar- 85 , Sadh ou Kilas, destaca-se na floricultura como um inibidor de crescimento de uso exclusivo em ornamentais, de fácil aplicação, sendo esta via foliar (BARRET, 1992; TINOCO et al., 2011) e já utilizado durante muitos anos por produtores na busca por plantas compactas (CANTLIFFE, 2009). Existem muitos trabalhos sobre a utilização de daminozide em ornamentais (CUQUEL; SABBAGH; OLIVEIRA, 2010; NEVES et al., 2009; BONACIN; RODRIGUES; MATTIUZ, 2006; MAINARDI; BELLÉ; MAINARDI, 2004; TOLOTTI; BELLÉ; MAINARDI, 2003), já que é o único retardante de crescimento registrado no Ministério da Agricultura para uso em culturas ornamentais no Brasil, porém poucas são as informações sobre seu uso em pimenteiras.

Vários fatores podem influenciar a altura e as respostas das plantas à aplicação de reguladores de crescimento, destacando-se a escolha do produto utilizado, que irá influenciar na escolha das concentrações, o método e a frequência de aplicação do produto, a espécie e o estágio de desenvolvimento da planta no momento da aplicação; as condições ambientais, como fotoperíodo e temperatura, e práticas culturais, como nutrição e o tipo de substrato utilizado no cultivo (DAVIS; STEFFENS; ANKHLA, 1988; GRZESIK, 1989; BARRET, 1992; MAINARDI; BELLÉ; MAINARDI, 2004; POBUDKIEWICZ, TREDER, 2006; NEVES et al., 2009; TINOCO et al., 2011). Assim, tendo em vista que os substratos possuem a função de promover suporte ao crescimento das plantas e regular a disponibilidade de nutrientes e água no meio, estes podem ser considerados fatores relevantes nas 
respostas das plantas à aplicação dos reguladores e no porte final esperado.

Os substratos agrícolas são definidos como um meio em que se desenvolvem as raízes das plantas cultivadas fora do solo, in situ (KÄMPF, 2000), podendo ser compostos por materiais que devem atender padrões de qualidade que independem de sua composição, como baixa densidade, elevada capacidade de troca catiônica, teor adequado de nutrientes, boa capacidade de retenção de água (BRUXEL et al., 2002), porosidade, drenagem e trocas gasosas suficiente para permitir uma boa aeração (KÄMPF, 2000), esterilidade quanto a presença patógenos e plantas daninhas (ANTUNES et al., 2002) e serem facilmente disponível na região do cultivo, sendo a fibra de côco ou areia possuidores de partes destas características e largamente utilizados na produção de ornamentais envasadas, visando com isso reduções nos seus custos.

Diante do exposto, objetivou-se avaliar o efeito do daminozide, em três pulverizações de diferentes concentrações, no crescimento e nas características morfofisiológicas das plantas de pimenta ornamental em vasos, contendo como substrato fibra de côco ou areia.

\section{Material e Métodos}

O experimento foi conduzido em casa de vegetação não-climatizada, coberto com telhas de fibra de vidro e laterais com tela de nylon, localizada no Departamento de Fitotecnia da Universidade Federal do Ceará, em Fortaleza-CE, situada a 19,5 $\mathrm{m}$ de altitude e coordenadas $3^{\circ} 44^{\prime} \mathrm{S}$ e $38^{\circ} 33^{\prime} \mathrm{W}$, durante o período de 11 de agosto a 2 de novembro de 2011, com temperatura e a umidade relativa do ar média de $34,45 \pm 3,22^{\circ} \mathrm{C}$ e $46,31 \pm 11,47 \%$ respectivamente, e luminosidade média de 600

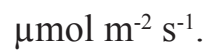

A cultivar de pimenta ornamental utilizada foi a Capsicum annuum cv. Treasures Red fornecida pela empresa Sakata Seed, que produz frutos cônicos de coloração vermelho brilhante ao amadurecer, posicionados de forma vertical nas folhagens que apresentam um verde profundo. Para a condução do experimento sementes de pimenta foram postas para germinar em bandejas de polietileno preto de 168 células contendo vermiculita - de granulométria mediana com partículas de 55-95\% > 2,4 mm como substrato.

Adotou-se delineamento inteiramente casualizado, com quatro repetições, arranjados em um esquema fatorial $5 \times 2$, constituído por cinco concentrações do regulador daminozide $(0,2,4,6$ e $8 \mathrm{~g} \mathrm{~L}^{-1}$ ) e dois tipos de substratos: fibra de côco (FC) granulada ou areia grossa. A unidade experimental foi constituída por cinco vasos, com uma planta por vaso. Plantas pulverizadas apenas com água destilada serviram como tratamento controle.

Os substratos utilizados, areia ou FC, foram adquiridos no comércio da região e caracterizados quimicamente pelo Laboratório de Química e Fertilidade do Departamento de Ciências do Solo/ Centro de Ciências Agrárias da Universidade Federal do Ceará, sendo as características físicas, capacidade retenção de água (CRA) e relação poros e sólidos $(\mathrm{P} / \mathrm{S})$, e químicas, potencial hidrogeniônico $(\mathrm{pH})$ e condutividade hidráulica (CE), realizadas de acordo com a metodologia proposta por Kämpf, Takane e Siqueira (2006) (Tabela 1).

Antes do plantio, vasos de plástico rígido do tipo polietileno preto, com drenos na extremidade inferior, apresentando $11,5 \mathrm{~cm}$ de altura, $14,5 \mathrm{~cm}$ de diâmetro superior, $10,5 \mathrm{~cm}$ de diâmetro inferior e capacidade de $1,15 \mathrm{~L}$, foram cheios com os substratos FC ou areia, sendo em seguida transplantadas mudas de pimenta com 17 dias após a semeadura (DAS). Foram realizadas três aplicações do fertilizando de lenta liberação Basacote $\AA 3$ 3M (15-9-12), a 10 g planta $^{-1}$, no momento a do transplantio, e a 5 g planta $^{-1}$, aos 33 e 46 DAS, devido às plantas apresentarem sintomas de sintomas de amarelecimento. Durante o experimento, adotou-se a rega manual das plantas com o auxílio de uma mangueira, uma vez ao dia, até a saturação total dos substratos. 
Tabela 1. Caracterização química e física dos substratos utilizados.

\begin{tabular}{|c|c|c|}
\hline \multirow{2}{*}{ Atributos } & \multicolumn{2}{|c|}{ Substratos } \\
\hline & Fibra de côco & Areia \\
\hline $\mathrm{Na}^{+}\left(\right.$cmolc $\left.\mathrm{Kg}^{-1}\right)$ & 3,87 & 0,01 \\
\hline $\mathrm{Zn}\left(\mathrm{mg} \mathrm{Kg}^{-1}\right)$ & 18,1 & 3,1 \\
\hline $\operatorname{Mn}\left(\mathrm{mg} \mathrm{Kg}{ }^{-1}\right)$ & 6,6 & 14,4 \\
\hline $\mathrm{Fe}\left(\mathrm{mg} \mathrm{Kg}^{-1}\right)$ & 17,2 & 48,1 \\
\hline $\mathrm{Cu}\left(\mathrm{mg} \mathrm{Kg}{ }^{-1}\right)$ & 0,7 & 0,4 \\
\hline $\mathrm{C} / \mathrm{N}$ & 10 & 13 \\
\hline $\mathrm{pH}$ & 5,8 & 6,5 \\
\hline $\mathrm{CE}\left(\mathrm{dS} \mathrm{m} \mathrm{m}^{-1}\right)$ & 0,41 & 0,22 \\
\hline $\left.\mathrm{P}_{2} \mathrm{O}_{5} \mathrm{mg} \mathrm{Kg}^{-1}\right)$ & 17 & 17 \\
\hline $\mathrm{K}_{2} \mathrm{O}\left(\mathrm{cmolc} \mathrm{Kg}^{-1}\right)$ & 6,17 & 0,03 \\
\hline $\mathrm{Ca}\left(\mathrm{cmolc} \mathrm{Kg}^{-1}\right)$ & 14,2 & 0,60 \\
\hline $\mathrm{Mg}\left(\mathrm{cmolc} \mathrm{Kg}^{-1}\right)$ & 12,6 & 0,60 \\
\hline $\mathrm{S}\left(\right.$ cmolc $\left.\mathrm{Kg}^{-1}\right)$ & 36,8 & 1,2 \\
\hline $\mathrm{N}\left(\mathrm{g} \mathrm{Kg}^{-1}\right)$ & 1,24 & 0,07 \\
\hline $\mathrm{C}\left(\mathrm{g} \mathrm{Kg}^{-1}\right)$ & 12,06 & 0,9 \\
\hline $\mathrm{MO}\left(\mathrm{g} \mathrm{Kg}^{-1}\right)$ & 10,79 & 1,56 \\
\hline $\mathrm{U}\left(\mathrm{g} 100 \mathrm{~g}^{-1}\right)$ & 0,83 & 0,17 \\
\hline $\mathrm{D}\left(\mathrm{g} \mathrm{L}^{-1}\right)$ & 65 & 1385 \\
\hline $\mathrm{CRA}\left(\mathrm{mL} \mathrm{g}^{-1}\right)$ & 335 & 310 \\
\hline $\mathrm{P} / \mathrm{S}$ & 4 & 0,79 \\
\hline
\end{tabular}

U - umidade; D - densidade aparente; CRA - capacidade de retenção de água; P/S - relação poros e sólidos; CE - condutividade elétrica.

Fonte: Elaboração dos autores.

Para o preparo das caldas do regulador, as concentrações testadas foram pesadas e ajustadas para 2,4; 4,8; 7,2 e 9,6 gramas do produto comercial B-nine e em seguidas diluídas em $1 \mathrm{~L}$ de água destilada $\left(\mathrm{pH}=4\right.$ e $\left.\mathrm{CE}=0,04 \mathrm{dS} \mathrm{m}^{-1}\right)$. Para as aplicações utilizou-se um pulverizador manual com capacidade de $2 \mathrm{~L}$, sendo as plantas pulverizadas de maneira uniforme, obtendo-se a cobertura completa das folhas e hastes. As concentrações testadas foram aplicadas aos 25, 40 e 55 DAS, sempre às 16 horas.

Aos 83 DAS foram avaliadas as variáveis altura da planta (AP; cm), realizada da base da planta rente ao substrato até seu ápice, com o uso de uma régua milimetrada, diâmetro do caule ( $\mathrm{DC} ; \mathrm{mm}$ ), medido a $3 \mathrm{~cm}$ da base da planta rente ao substrato, largura (LFR; mm) e comprimento dos frutos (CFR; $\mathrm{mm}$ ) com o uso de paquímetro digital, número de frutos por planta (NFR), índice relativo de clorofila
(IRC), área foliar $\left(\mathrm{AF} ; \mathrm{cm}^{2}\right)$, trocas gasosas foliares das plantas, massa seca das folhas (MSF; g), caule (MSC; g), frutos (MSF; g) e raiz (MSR; g).

Para avaliação do IRC utilizou-se o método indireto através do medidor portátil de clorofila Minolta, SPAD-502, sendo as leituras feitas em três folhas formadas e totalmente dispostas à luz solar, localizadas no terço superior de cada planta. Para a estimativa da área foliar das plantas utilizou-se um medidor de superfície (LI - 3100, Area Meter, Li-Cor., Inc., Lincoln, Nebraska, USA). Para as trocas foliares utilizou-se o analisador portátil de $\mathrm{CO}_{2}$ por radiação infra-vermelho ("Infra Red Gas Analyser-IRGA", modelo LCi System, ADC, Hoddesdon, UK) sendo obtidos a temperatura da folha (Tl), concentração interna de $\mathrm{CO}_{2}(\mathrm{Ci})$, taxa de transpiração $(\mathrm{E})$, condutância estomática (gs), fotossíntese líquida (A). As leituras foram feitas 
em cinco plantas por tratamento em folhas adultas e totalmente expandidas do seu terço superior. As medições ocorreram entre 8:00 e 11:00 horas, utilizando-se uma fonte de radiação artificial (cerca de $\left.1600 \mathrm{mmol} \mathrm{m}^{-2} \mathrm{~s}^{-1}\right)$.

Após todas estas avaliações, para a obtenção das variáveis de massa seca, as plantas foram separadas em folhas, caules, frutos e sistema radicular e acondicionadas em sacos de papel para serem secas em estufa com circulação forçada de ar, a $70^{\circ} \mathrm{C}$ por $72 \mathrm{~h}$, até peso constante.

Os dados foram submetidos à análise de variância utilizando o programa estatístico Sisvar ${ }^{\circledR}$. Para efeito significativo entre os tipos de substratos as médias foram comparadas pelo teste de Tukey a $5 \%$ de probabilidade. Para avaliação entre concentrações de daminozide e interação entre os fatores utilizou-se a análise de regressão, sendo a escolha dos modelos baseadas na significância dos coeficientes linear e quadrático, por meio do teste $\mathrm{t}$ de "Student", aos níveis de 1 e $5 \%$ de probabilidade.

\section{Resultados e Discussão}

Verificou-se interação significativa entre os fatores estudados (tipo de substrato e concentração de daminozide) apenas para as variáveis altura da planta (AP), massa seca do caule (MSC) e frutos (MSFR), número (NFR) e largura de frutos (LFR), índice relativo de clorofila (IRC), área foliar (AF) e condutância estomática (gs) (Tabela 2).

Tabela 2. Análise de variância das variáveis analisadas nas plantas de pimenta ornamental aos 83 dias após a semeadura.

\begin{tabular}{lcccccccc}
\hline \multirow{2}{*}{ FV } & \multicolumn{7}{c}{ Quadrados médios } \\
\cline { 2 - 8 } & $\mathbf{A P}$ & $\mathbf{D C}$ & $\mathbf{N F R}$ & $\mathbf{C F R}$ & $\mathbf{L F R}$ & MSFR & MSF & MSC \\
\hline Substrato & $73,1^{* *}$ & $37,00^{* *}$ & $3101,1^{* *}$ & $13,2^{* *}$ & $19,7^{* *}$ & $166,5^{*}$ & $66,8^{* *}$ & $29,10^{* *}$ \\
Daminozide & $98,4^{* *}$ & $0,91^{* *}$ & $3122,1^{* *}$ & $408,1^{* *}$ & $39,6^{* *}$ & $130,6^{* *}$ & $0,4^{\text {ns }}$ & $0,06^{\text {ns }}$ \\
S $\times \mathbf{D}$ & $3,11^{* *}$ & $0,12^{\text {ns }}$ & $550,6^{* *}$ & $6,2^{\text {ns }}$ & $6,6^{* *}$ & $28,8^{* *}$ & $0,2^{\text {ns }}$ & $0,5^{* *}$ \\
Resíduo & 0,36 & 0,07 & 59,90 & 2,4 & 0,9 & 1,2 & 0,1 & 0,0 \\
CV (\%) & 5,3 & 3,9 & 27,4 & 10,8 & 9,7 & 22,7 & 6,8 & 8,9 \\
\hline \multicolumn{1}{c}{$\mathbf{F V}$} & $\mathbf{M S R}$ & $\mathbf{I R C}$ & $\mathbf{A F}$ & $\mathbf{g s}$ & $\mathbf{C i}$ & $\mathbf{E}$ & $\mathbf{T l}$ & $\mathbf{A}$ \\
\hline Substrato & $17,0^{* *}$ & $54,26^{* *}$ & $1111829,0^{* *}$ & $2,60^{* *}$ & $626,58^{*}$ & $0,62^{\text {ns }}$ & $0,38^{\text {ns }}$ & $0,14^{\text {ns }}$ \\
Daminozide & $2,1^{* *}$ & $356,97^{* *}$ & $174177,5^{* *}$ & $1,16^{* *}$ & $0,37^{\text {ns }}$ & $0,68^{*}$ & $0,47^{\text {ns }}$ & $11,15^{*}$ \\
S $\times$ D & $0,3^{\text {ns }}$ & $32,30^{* *}$ & $193446,7^{* *}$ & $0,60^{* *}$ & $116,18^{\text {ns }}$ & $0,21^{\text {ns }}$ & $0,12^{\text {ns }}$ & $2,45^{\text {ns }}$ \\
Resíduo & 0,2 & 5,7 & 12644,6 & 0,13 & 113,08 & 0,20 & 0,89 & 2,92 \\
CV (\%) & 14,93 & 3,36 & 12,10 & 31,33 & 3,96 & 9,54 & 2,94 & 9,98 \\
\hline
\end{tabular}

AP - altura da planta; DC - diâmetro do caule; NFR - número de frutos; CFR - comprimento dos frutos; LFR - largura dos frutos; MSFR - massa seca dos frutos; MSF - massa seca das folhas; MSC - massa seca caule; MSR - massa seca raiz; IRC - índice relativo de clorofila; $\mathrm{AF}$ - área foliar; $\mathrm{A}$ - fotossíntese liquida; gs - condutância estomática; $\mathrm{Ci}$ - concentração interna de $\mathrm{CO}_{2}$; $\mathrm{Tl}$ - temperatura da folha.

${ }^{*} \mathrm{e}^{* *}$ Significativo a 0,05 e a 0,01 de probabilidade, respectivamente; ${ }^{\text {ns }}$ - não significativo pelo teste $\mathrm{F}$.

Fonte: Elaboração dos autores.

Em relação ao efeito isolado do fator substratos, observou-se que plantas de pimenta ornamental cultivadas no substrato fibra de côco (FC) apresentaram-se com caule mais espessos $(7,47$ $\mathrm{mm})$, maior massa seca de folhas $(5,88 \mathrm{~g})$, raízes $(4,19 \mathrm{~g})$, com superior concentração interna de $\mathrm{CO}_{2}$ $(271,76 \mathrm{ppm})$ e taxa de transpiração $(4,85 \mathrm{mmol}$ $\mathrm{m}^{-2} \mathrm{~s}^{-1}$ ) equivalente a uma superioridade de 25,7; 44,$0 ; 68,2 ; 2,6$ e 4,5\%, respectivamente, quando comparadas com as plantas cultivadas na areia, caules mais espessos $(7,47 \mathrm{~mm})$, maior massa seca de folhas $(5,88 \mathrm{~g})$ e raízes $(4,19 \mathrm{~g})$ (Tabela 3). Já para as variáveis referentes à fotossíntese líquida (A) e temperatura das folhas (Tl) não houveram 
diferenças significativas entre substratos (Tabela fotossintética e de transpiração das plantas de

2) inferindo-se que diferenças químicas e físicas

pimenta ornamental (Tabela 3).

(Tabela 1) não foram capazes de diminuir as taxa

Tabela 3. Efeito isolado dos substratos fibra de côco e areia nas plantas de pimenta ornamental aos 83 dias após a semeadura.

\begin{tabular}{|c|c|c|c|c|c|c|c|}
\hline \multirow{2}{*}{ Substratos } & \multirow{2}{*}{$\begin{array}{c}\mathrm{DC} \\
(\mathrm{mm})\end{array}$} & \multirow{2}{*}{$\begin{array}{c}\text { MSF } \\
\text { (g) }\end{array}$} & \multirow{2}{*}{$\begin{array}{c}\text { MSR } \\
\text { (g) }\end{array}$} & \multirow{2}{*}{$\begin{array}{c}\mathbf{C i} \\
(\mathbf{p p m})\end{array}$} & $\mathbf{E}$ & A & \multirow{2}{*}{$\begin{array}{c}\text { Tl } \\
\left({ }^{\circ} \mathrm{C}\right)\end{array}$} \\
\hline & & & & & \multicolumn{2}{|c|}{$\left(\mathrm{mmol} \mathrm{m} \mathrm{m}^{-2} \mathrm{~s}^{-1}\right)$} & \\
\hline $\mathrm{FC}$ & $7,47 \mathrm{a}$ & $5,88 \mathrm{a}$ & $4,19 \mathrm{a}$ & $271,76 \mathrm{a}$ & $4,85 \mathrm{a}$ & $17,07 \mathrm{a}$ & $32,28 \mathrm{a}$ \\
\hline Areia & $5,55 \mathrm{~b}$ & $3,29 \mathrm{~b}$ & $2,86 \mathrm{~b}$ & $264,68 \mathrm{~b}$ & $4,63 \mathrm{~b}$ & $17,17 \mathrm{a}$ & $32,10 \mathrm{a}$ \\
\hline $\mathrm{CV}(\%)$ & 3,94 & 6,80 & 6,80 & 3,96 & 9,54 & 9,98 & 2,94 \\
\hline
\end{tabular}

$\mathrm{FC}$ - fibra de côco, DC - diâmetro do caule, MSF - massa seca folhas, $\mathrm{MSR}$ - massa seca raiz, $\mathrm{Ci}$ - concentração interna $\mathrm{CO}_{2}$ nas folhas, $\mathrm{E}$ - taxa de transpiração, A - fotossíntese líquida, Tl - temperatura da folha

*Médias seguidas da mesma letra na coluna não diferem entre se pelo teste de Tukey a 5\% de probabilidade

Fonte: Elaboração dos autores.

O baixo desenvolvimento das plantas de pimenta em substratos inorgânicos como a areia deve-se segundo Loach (1998) ao fato de possuírem poucas ou nenhuma reservas de nutrientes, sendo necessária uma complementação nutricional adequada e eficiente durante o crescimento da cultura. Diante da análise química e física dos substratos (Tabela 1) percebe-se que a FC apresentou maiores quantidade de macro e micronutrientes assim como maior teor de matéria orgânica e capacidade de retenção de água o que favoreceu o crescimento e desenvolvimento das plantas neste substrato. A particular estrutura física e química da FC como substrato (Tabela1) torna-a adequada para a produção de plantas em vasos e dificilmente superável por outro tipo de material, seja de origem mineral ou orgânica, na produção e no cultivo de hortaliças e flores sem o uso do solo (CARRIJO; LIZ; MAKISHIMA, 2002). Embora não se tenha feita a correlação entre características físicas dos substratos versus a liberação do fertilizante, diante da caracterização dos mesmos percebe-se o substrato areia por apresentar baixa CRA, porosidade e alta densidade, indica ser um meio com maior retenção de calor, podendo este fator estar diretamente ligado à baixa disponibilidade do basocote para as planta provocando seu subdesenvolvimento. O que corrobora com Silva, Amaral e Castilho (2013) onde citam que a taxa de liberação dos nutrientes pelos grânulos de fertilizante é diretamente proporcional à temperatura do solo ou substrato, uma vez que a temperatura promove expansão da camada de resina, provocando aumento de sua permeabilidade à água e rápida dissolução.

Quanto as características químicas de acordo com Cavins et al. (2000), apenas o substrato FC apresentou valores adequados de $\mathrm{CE}$, no qual indicam uma faixa entre 0,36 a $0,89 \mathrm{dS} \mathrm{m}^{-1}$ como um padrão tolerado para a maioria das plantas em crescimento. Em relação ao pH segundo Kämpf (2000) a faixa ideal encontra-se entra que 5,0 a 5,8 para substratos orgânicos e de 6,0 a 6,5 para substratos inorgânicos, estando a FC e a areia, respectivamente, dentro da faixa tolerada pela cultura da pimenta (Tabela 1).

Para as variáveis DC, MSR, comprimento dos frutos (CFR), A e E verificou-se efeito isolado do regulador, notando que a MSF não foi afetada pelo tratamento com diferentes concentrações de daminozide(Tabela2). Soboefeito das concentrações observou-se para a variável DC um comportamento quadrático notando a partir da concentração $2 \mathrm{~g} \mathrm{~L}^{-1}$ um aumento na espessura dos caules das plantas tratadas o que tornou estes mais espessos que os 
caules das plantas controle atingindo ponto de máxima a 5,33 $\mathrm{g} \mathrm{L}^{-1}$, o que equivale a caules de 6,80 mm de diâmetro (Tabela 4), efeito este favorável em plantas ornamentais uma vez que hastes finas podem prejudicar a sustentação de frutos e inflorescências. Kofidis, Giannakoula e Ilias (2008) ao utilizarem as concentrações 0,5 ou $1 \mathrm{~g} \mathrm{~L}^{-1}$ de daminozide também obtiveram plantas de coentro com caules mais espessos, fortes e compactos justificando ser devido ao aumento de volume do tecido colênquima e o aumento no número de vasos nos feixes vasculares. Já Cuquel, Sabbagh e Oliveira (2010) relatam a não interferência do regulador para este mesmo parâmetro nas hastes de girassol ornamental.

Tabela 4. Efeito isolado do fator concentrações de daminozide nas plantas de pimenta aos 83 dias após semeadura.

\begin{tabular}{|c|c|c|c|c|c|c|c|}
\hline \multirow{2}{*}{ Variáveis } & \multicolumn{5}{|c|}{ Concentrações $\left(\mathrm{g} \mathrm{L}^{-1}\right)$} & \multirow{2}{*}{ Equações } & \multirow{2}{*}{$\mathbf{R}^{2}$} \\
\hline & $\mathbf{0}$ & 2 & 4 & 6 & 8 & & \\
\hline DC & 5,9 & 6,5 & 6,7 & 6,7 & 6,6 & $Y=5,96+0,32^{* *} x-0,03^{* *} x^{2}$ & 0,99 \\
\hline CFR & 26,3 & 14,9 & 12,2 & 4,5 & 8,7 & $Y-22,45-2,03^{* *} x$ & 0,80 \\
\hline MSF & 4,3 & 5,0 & 4,5 & 4,6 & 4,5 & $Y=4,6$ & - \\
\hline MSR & 2,7 & 3,7 & 4,0 & 3,9 & 3,3 & $\mathrm{Y}=2,77+0,56^{* *} \mathrm{x}-0,06^{* *} \mathrm{x}^{2}$ & 0,98 \\
\hline $\mathbf{E}$ & 5,0 & 5,0 & 4,7 & 4,5 & 4,4 & $Y=5,07-0,08^{* *} x$ & 0,96 \\
\hline $\mathbf{A}$ & 17,9 & 18,3 & 17,2 & 16,1 & 16,0 & $\mathrm{Y}=18,33-0,30^{* *} \mathrm{x}$ & 0,82 \\
\hline
\end{tabular}

DC - diâmetro do caule (mm); CFR - comprimento dos frutos (mm); MSF - massa seca folhas (g); MSR - massa seca raiz (g); $\mathrm{E}$ - taxa de transpiração $\left(\mathrm{mmol} \mathrm{m} \mathrm{m}^{-2} \mathrm{~s}^{-1}\right) ; \mathrm{A}$ - fotossíntese liquida $\left(\mathrm{mmol} \mathrm{m} \mathrm{m}^{-2} \mathrm{~s}^{-1}\right)$.

${ }^{*}$ e ${ }^{* *}$ Significativo a 0,05 e a 0,01 de probabilidade, respectivamente; ns - não significativo pelo teste $\mathrm{F}$.

Fonte: Elaboração dos autores.

A MSF não foi influenciada pela ação do regulador, já para MSR notou-se comportamento quadrático com ponto de máxima em plantas tratadas com 4,56 $\mathrm{g} \mathrm{L}^{-1}$ atingindo 4,04 g (Tabela 4). Os efeitos de reguladores vegetais na massa seca das plantas são relatados por alguns autores, com respostas variando de acordo com a espécie, dosagem e frequência utilizada. Grossi et al. (2005) verificaram reduções lineares na massa seca das plantas de pimenta ornamental cv. Pitanga tratadas com paclobutrazol, aplicado tanto via foliar como via substrato. Bonacin, Rodrigues e Mattiuz (2006) verificaram reduções na massa seca de caule, pecíolos e lâminas de híbridos de girassol ornamental quando utilizaram soluções de paclobutrazol $(0,003$ g vaso $\left.{ }^{-1}\right)$, cloreto de chlormequat $\left(0,4 \mathrm{~g} \mathrm{vaso}^{-1}\right) \mathrm{e}$ daminozide $\left(0,5 \mathrm{~g} \mathrm{vaso}^{-1}\right)$.

Referentes às trocas gasosas fotossíntese liquida (A) e taxa de transpiração (E) nas plantas de pimenta verificou-se comportamento linear negativo nas equações com o aumento das concentrações de daminozide (Tabela 4), com reduções máxima de 13,24\% para fotossintética liquida e de $12,97 \%$ para a taxa de transpiração das plantas ao serem tratadas com $8 \mathrm{~g} \mathrm{~L}^{-1}$ (Tabela 3), corroborando com Roberts e Domir (1983) que, ao utilizarem $5 \mathrm{~g} \mathrm{~L}^{-1}$ de daminozide no caule de mudas Platanus occidentalis L variedades americano e $P$. occidentalis $\mathrm{L}$ var. bordo prata, também verificaram declínios na fotossintética liquida destas plantas. Já Ferree e Hall (1978) relatam que uma única aplicação de $2 \mathrm{~g} \mathrm{~L}^{-1}$ de daminozide não influenciou a fotossintética liquida de árvores de maçã cultivadas em casa de vegetação.

Para as variáveis que apresentaram interações significativas entre substratos e daminozide (Tabela 2) percebe-se que plantas na FC apresentaramse mais altas em $23,39 \%$ em relação às plantas na areia. Plantas mais desenvolvidas, vigorosas e de maior altura na FC podem estar relacionada à superioridade química e física deste substrato em relação à areia, destacando-se por possuir maior 
capacidade de retenção de água, porosidade e baixa densidade, características estas fundamentais para o desenvolvimento das plantas (Tabela 1). Frente a relação $\mathrm{P} / \mathrm{S}$ igual a 4 para a $\mathrm{FC}$, Kämpf, Takane e Siqueira (2006) dizem que substratos agrícolas devem possuir esta acima de 3, o que reforça a viabilidade e superioridade deste material (Tabela 1).

A partir da menor concentração de daminozide, plantas de pimenta apresentaram reduções em altura, verificando-se comportamento quadrático e ponto de mínimo nas curvas, com máxima redução em altura, quando as plantas foram tratadas com 7,48 $\mathrm{g} \mathrm{L}^{-1}$ na FC e $8 \mathrm{~g} \mathrm{~L}^{-1}$ na areia, atingindo altura de 9,41 cm e 6,02 cm, respectivamente (Tabela 5). Já Aloni e Pashkar (1987) também trabalhando com daminozide na espécie Capsicum annuum alcançaram máxima redução em altura ao utilizar a $5 \mathrm{~g} \mathrm{~L}^{-1}$, o que corroboraram com os resultados de Pisarczyk e Splittstoesser (1979), que também verificaram reduções na altura de plântulas de tomateiro tratadas com daminozide. Já em girassol ornamental cv. BRS Oasis, Cuquel, Sabbagh e Oliveira (2010) citam reduções em altura na concentração $6 \mathrm{~g} \mathrm{~L}^{-1}$ aplicadas aos 15 e 30 DAS, ressaltando que a melhor para reduzir a altura das plantas depende simultaneamente da concentração e do número de aplicações. Resultados similares a este foi observado por Watanabe (2007) com girassol cultivar 'Pacino' ao controlar a altura das plantas aplicando daminozide a $4 \mathrm{~g} \mathrm{~L}^{-1}$.

Definição de altura em plantas ornamentais, como nas pimenteiras, torna-se um parâmetro difícil de definir, podendo este variar de acordo com o gosto do consumidor, embora já exista padrões de qualidade para sua comercialização já determinado pela Cooperativa Velling de Holambra.

Quanto ao número (NFR) e largura dos frutos (LFR) verificou-se que plantas na FC apresentaram maior produtividade $\left(67,0\right.$ frutos planta $\left.^{-1}\right)$ e frutos mais $\operatorname{largos}(13,15 \mathrm{~mm})$ em relação às plantas na areia. Frente à interação entre os fatores verificouse para o NFR um comportamento linear negativo em suas equações com reduções quanto ao NFR à medida que houve o aumento das concentrações de daminozide, notando maiores reduções nas plantas cultivadas na FC, com queda de 7,4 frutos a cada $1 \mathrm{~g}$ de daminozide, enquanto na areia essa queda foi de 3,8 frutos a cada $1 \mathrm{~g}$ do produto. Em relação à $L F$ verificou-se que plantas tratadas com $8 \mathrm{~g} \mathrm{~L}^{-1}$ apresentaram frutos de menor largura correspondendo a $7,75 \mathrm{~mm}$ na $\mathrm{FC}$ e $6,12 \mathrm{~mm}$ na areia (Tabela 5).

Percebe-se que a partir da menor concentração testada, daminozide foi capaz de reduzir a produção dos frutos de pimenta, bem como sua largura e comprimento, causando consequentes atrasos na floração das plantas de pimenta, afetando negativamente $\mathrm{o}$ aspecto final, característica esta indesejável para ornamentação. Tinoco et al. (2011), utilizando daminozide em plantas de gerânio, não observaram sua interferência quanto ao número de inflorescências por planta, porém citam que a partir de 2,5 $\mathrm{g} \mathrm{L}^{-1} \mathrm{o}$ diâmetro destas foram reduzidos prejudicando assim o aspecto final. Kofidis, Giannakoula e Ilias (2008), ao utilizarem $0,5 \mathrm{~g} \mathrm{~L}^{-1}$ de daminozide, também notaram reduções significativas no rendimento, produção nos frutos e floração de plantas de coentro corroborando com o observado no presente estudo, enquanto Appezzato e Castro (1982) citam que $3 \mathrm{~g} \mathrm{~L}^{-1}$ de daminozide não afetaram o número e peso total dos frutos de tomate. Já Grossi et al. (2005) relatam que pimentas ornamentais cv. Pitanga tratadas paclobutrazol, apresentaram frutos mais largos e com massa seca inalterada, citando que em outro experimento plantas de tomateiros ornamentais tratados com o mesmo regulador, apresentaram frutos com largura ligeiramente inferior aos frutos das plantas controle, levando a inferir que os efeitos dos reguladores variam de acordo com a espécie e as condições ambientais de cultivo. 
Tabela 5. Efeito da interação entre os fatores substratos e concentrações de daminozide nas plantas de pimenta ornamental aos 83 dias após semeadura.

\begin{tabular}{|c|c|c|c|c|c|c|c|}
\hline \multirow{2}{*}{ Variáveis } & \multicolumn{5}{|c|}{ Concentrações $\left(\mathrm{g} \mathrm{L}^{-1}\right)$} & \multirow{2}{*}{ Equações } & \multirow{2}{*}{$\mathbf{R}^{2}$} \\
\hline & 0 & 2 & 4 & 6 & 8 & & \\
\hline \multicolumn{8}{|l|}{$\mathbf{A P}$} \\
\hline Fibra & 18,8 & 13,1 & 11,5 & 10,6 & 9,0 & $Y=18,3-2,39^{* *} x+0,16^{* *} x^{2}$ & 0,95 \\
\hline Areia & 14,8 & 11,8 & 9,8 & 7,2 & 6,2 & $Y=-14,7-1,67^{* *} x+0,08^{* *} x^{2}$ & 0,98 \\
\hline \multicolumn{8}{|l|}{ NFR } \\
\hline Fibra & 52,4 & 74,5 & 36,1 & 16,1 & 7,3 & $\mathrm{Y}=67,03-7,43^{* *} \mathrm{x}$ & 0,75 \\
\hline Areia & 31,1 & 31,1 & 21,9 & 4,8 & 5,8 & $\mathrm{Y}=34,3-3,84^{* *} \mathrm{x}$ & 0,87 \\
\hline \multicolumn{8}{|l|}{ LFR } \\
\hline Fibra & 13,3 & 11,1 & 11,0 & 9,5 & 7,3 & $Y=13,16-0,68^{* *} x$ & 0,94 \\
\hline Areia & 11,7 & 11,3 & 8,9 & 8,1 & 5,7 & $\mathrm{Y}=12,22-0,76^{* *} \mathrm{x}$ & 0,95 \\
\hline \multicolumn{8}{|l|}{ MSFR } \\
\hline Fibra & 15,7 & 10,3 & 4,5 & 3,0 & 1,3 & $\mathrm{Y}=14,21-1,80^{* *} \mathrm{x}$ & 0,92 \\
\hline Areia & 5,9 & 4,5 & 1,9 & 1,3 & 1,1 & $\mathrm{Y}=5,48-0,64^{* *} \mathrm{x}$ & 0,89 \\
\hline \multicolumn{8}{|l|}{ MSC } \\
\hline Fibra & 3,5 & 2,8 & 2,6 & 2,8 & 3,0 & $Y=4,47-0,36^{* *} x+0,04^{* *} x^{2}$ & 0,95 \\
\hline Areia & 1,0 & 1,3 & 1,4 & 1,3 & 1,3 & $\mathrm{Y}=1,01+0,17^{* *} \mathrm{x}-0,02^{* *} \mathrm{x}^{2}$ & 0,83 \\
\hline \multicolumn{8}{|c|}{ 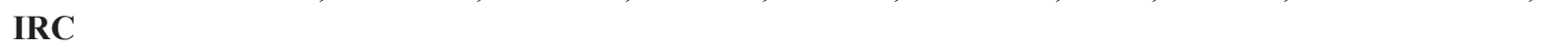 } \\
\hline Fibra & 54,0 & 66,7 & 71,5 & 72,7 & 74,4 & $\mathrm{Y}=54,8+5,98^{* *} \mathrm{x}-0,46^{* *} \mathrm{x}^{2}$ & 0,97 \\
\hline Areia & 62,5 & 66,7 & 75,2 & 74,2 & 72,4 & $Y=61,65+4,44^{* *} x-0,38^{* *} x^{2}$ & 0,91 \\
\hline \multicolumn{8}{|c|}{$x^{2}$} \\
\hline Fibra & 1461,5 & 1286,0 & 1001,1 & 990,0 & 739,4 & $Y=1444-87,06^{* *} x$ & 0,95 \\
\hline Areia & 623,7 & 869,9 & 788,4 & 861,5 & 668,4 & $\mathrm{Y}=642,7+107,4^{* *} \mathrm{x}-12,9^{* *} \mathrm{x}^{2}$ & 0,76 \\
\hline \multicolumn{8}{|c|}{ 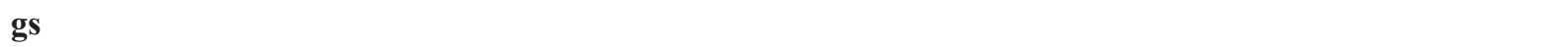 } \\
\hline Fibra & 2,1 & 1,9 & 1,1 & 0,9 & 1,0 & $Y=2,24-0,34^{* *} x+0,02 x^{2}$ & 0,92 \\
\hline Areia & 1,1 & 1,0 & 1,1 & 0,8 & 0,8 & $Y=1,08-0,02 x-0,002 x^{2}$ & 0,70 \\
\hline
\end{tabular}

$\mathrm{AP}$ - altura da planta (cm); NFR - número de frutos; LFR - largura dos frutos (mm); MSFR - massa seca dos frutos (g); MSC massa seca caule (g); IRC - índice relativo de clorofila (spad); $\mathrm{AF}$ - área foliar $\left(\mathrm{cm}^{2}\right)$; gs - condutância estomática $\left(\mathrm{mmol} \mathrm{m} \mathrm{m}^{-2} \mathrm{~s}^{-1}\right)$. ${ }^{*} \mathrm{e}^{* *}$ Significativo a 0,05 e a 0,01 de probabilidade, respectivamente.

Fonte: Elaboração dos autores.

Para MSC e MSFR verificou-se maior produção nas plantas cultivadas na FC apresentando superioridade de $72,6 \%$ e $62,4 \%$, respectivamente, quando comparadas com a areia. Sob o efeito das concentrações notou-se para MSC comportamento quadrático para ambos os substratos, com ponto mínimo na curva alcançado na concentração 4,64 $\mathrm{g} \mathrm{L}^{-1}$ para a $\mathrm{FC}$ e máximo a 4,82 $\mathrm{g} \mathrm{L}^{-1}$ na areia. Frente às reduções observadas à medida que houve o aumento das concentrações de daminozide tanto para número, largura e comprimento dos frutos, estas refletiram diretamente em sua MSFR que foi reduzida de forma linear negativa em ambos os substratos, com um equivalente de $1,80 \mathrm{~g}$ nos frutos das plantas cultivadas na FC e de 0,63 g quando cultivadas na areia, a cada aumento unitário nas concentrações do regulador (Tabela 5).

Através da partição de massa seca pode-se comprovar as significativas mudanças na produção e alocação de fotoassimilados das plantas ao longo dos tratamentos com daminozide, notando semelhante comportamento entre os substratos. Verifica-se que com aumento das concentrações houve drásticas reduções na produção na partição de MSFR, de 54,38 a $9,47 \%$ na $\mathrm{FC}$ e 51,47 a $13,17 \%$ na areia, com consequentes aumentos na de MSC, MSF e MSR. Tais aumentos nesta distribuição podem ter sido estimulados em conseqüência da inibição do 
crescimento reprodutivo das plantas, evidenciando com isso o forte efeito deletério do produto na frutificação das pimenteiras e sua possível interferência na distribuição de carboidratos nos diferentes órgãos (Figura 1). Albuquerque, Mouco e Albuquerque Neto (2008) destacam que videiras ao serem tratadas com $3 \mathrm{~g} \mathrm{~L}^{-1}$ de daminozide, além de crescerem menos, também tiveram menor acúmulo de massa seca, não favoreceu o aumento nas concentrações de nutrientes nos ramos e pecíolos e a formação de gemas férteis e panículas florais, afetando diretamente o acúmulo de carboidratos por estas plantas.

Figura 1. Partição de massa seca entre folha, caule, frutos e raízes das plantas de pimenta ornamental em função das concentrações de daminozide cultivadas em fibra de côco (A) e areia (B).
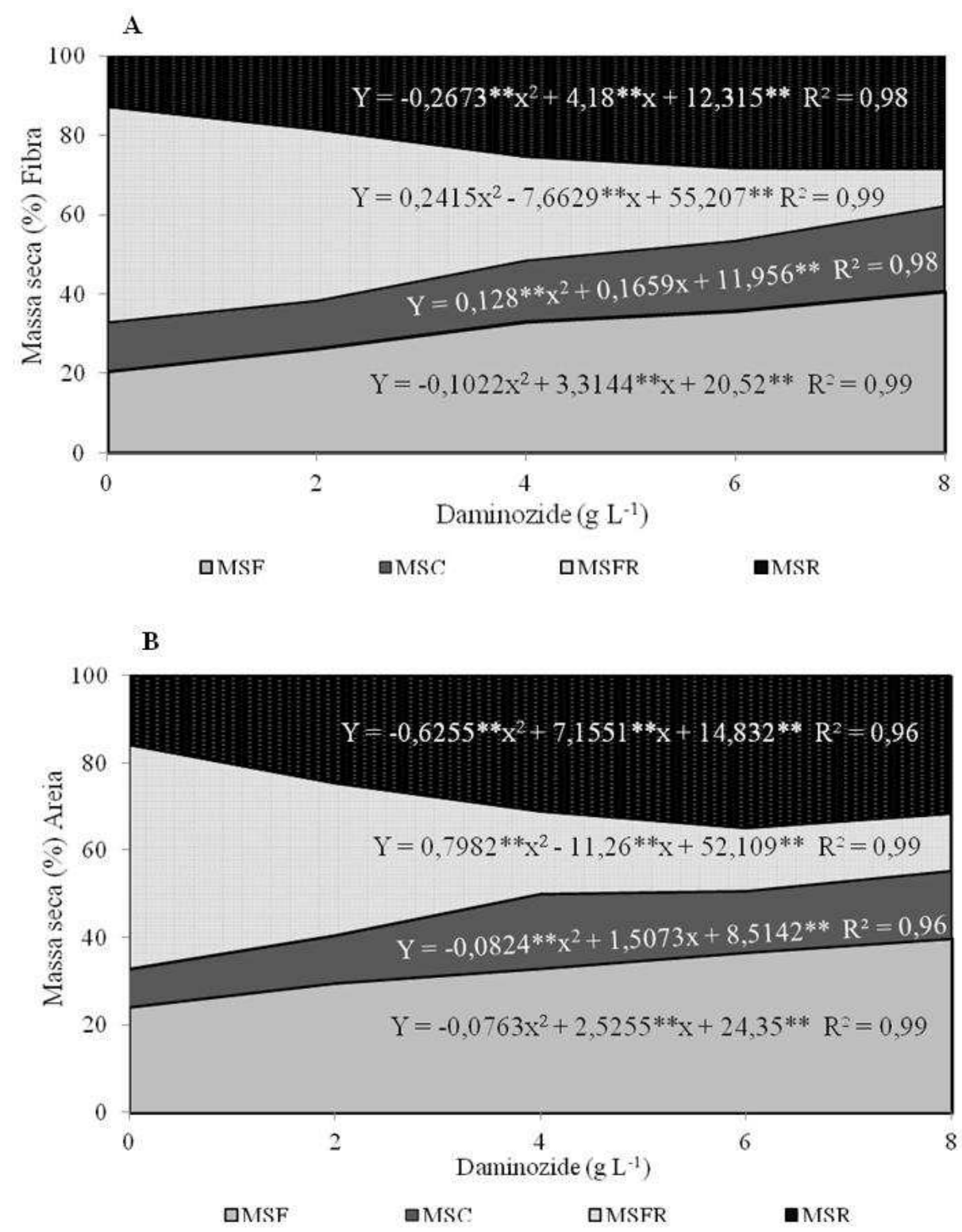

${ }^{*}$ e ${ }^{* *}$ Significativo a 0,05 e a 0,01 de probabilidade, respectivamente.

Fonte: Elaboração dos autores. 
Frente à interação significativa para o índice relativo de clorofila (IRC) nas folhas de pimenta, pode-se perceber um aumento nos valores ao longo das concentrações de daminozide, em ambos os substratos, ajustando-se a um comportamento quadrático em suas equações com pontos ótimos na concentração $6,56 \mathrm{~g} \mathrm{~L}^{-1}$ na $\mathrm{FC}$ e 5,77 $\mathrm{g} \mathrm{L}^{-1}$ na areia (Tabela 5) deixando-as com um verde mais intenso, de aparência mais atrativa e contrastante entre o verde das folhas e o vermelho dos frutos. Kofidis, Giannakoula e Ilias (2008) ao utilizarem 0,5 $\mathrm{g} \mathrm{L}^{-1}$ de daminozide em plantas de coentro perceberam que suas folhas apresentaram 16\% mais conteúdo de clorofila em relação às plantas controle, porém percebendo redução de $13 \%$ neste conteúdo quando tratadas com $1 \mathrm{~g} \mathrm{~L}^{-1}$ do produto. Grossi et al. (2005) também notou aumentos no IRC nas folhas de Capsicuum annuum cv. Pitanga ao serem tratadas com paclobutrazol, com aumentos lineares no teor de clorofila notando folhas de coloração verde mais intensa, corroborando com Aloni e Pashkar (1987) em Capsicuum annuum tratadas com 0,001 ou $0,005 \mathrm{mg} \mathrm{L}^{-1}$ do mesmo regulador. Mesmo diante destes relatos não é claro se o aumento no IRC pelos reguladores deve-se a biossíntese da clorofila reforçada ou simplesmente um efeito “concentração" devido à redução na expansão foliar (DAVIS; STEFFENS; ANKHLA, 1988).

Quanto à área foliar (AF) verificou-se interação significativa entre substratos e concentrações de daminozide, em que plantas no substrato FC apresentaram maiores áreas foliares, o que era de se esperar uma vez que a FC apresentou superioridade em atributos químicos e físicos como: maior teor de nutrientes, maior CRA e relação $\mathrm{P} / \mathrm{S}$, respectivamente (Tabela 1). Quando analisada dentro das concentrações do regulador verificouse comportamento linear negativo na FC com reduções em AF com o aumento das concentrações do regulador, com redução máxima de 49,4\% na concentração $8 \mathrm{~g} \mathrm{~L}^{-1}$ (Tabela 5). Já no substrato areia houve um comportamento quadrático com ponto máximo na concentração $4,15 \mathrm{~g} \mathrm{~L}^{-1}$ proporcionando plantas de AF igual a $866,09 \mathrm{~cm}^{2}$. Diante dos efeitos do regulador na AF das plantas, percebe-se que a baixa taxa de transpiração observada nas plantas (Tabela 3) pode estar diretamente correlacionada a esta variável, indicando possivelmente que a perda de água foi reduzida porque havia menos área para a intercepção da luz e energia necessária para impulsionar o processo de transpiração. Tais verificações estão de acordo com as pesquisas conduzidas nos EUA que demonstraram que plantas tratadas com determinados reguladores de crescimento apresentaram reduções em sua taxa de transpiração devido à diminuições em superfície foliar, o que também proporcionou as mesmas uma maior resistência ao estresse hídrico (MARCUM; JIANG, 1997; JIANG; FRY, 1998).

Uma vez que as folhas representam um importante papel estético no preenchimento dos espaços para cobertura dos vasos e os frutos como sendo o principal produto das pimentas, reduções em área foliar acompanhadas por reduções no número de frutos observadas quando as mesmas foram submetidas às aplicações de daminozide, tornam-se grandes inconvenientes na sua utilização como planta ornamental para vaso. Relatos sobre reduções em área foliar provocados por reguladores também foram verificados por Mainardi, Bellé e Mainardi (2004) que ao aumentar as frequências de aplicações das concentrações de daminozide verificaram reduções na área foliar em crisântemo afetando seu aspecto ornamental; Silva et al. (1980) em begônia, onde citam que daminozide a $2 \mathrm{~g} \mathrm{~L}^{-1}$ chegou a reduzir $76 \%$ a área foliar e Aloni e Pashkar (1987) em Capsicuum annuum utilizando 0,001 ou $0,005 \mathrm{mg} \mathrm{L}^{-1}$ do regulador paclobutrazol. De acordo com Barret (1992) os inibidores de giberelina, como daminozide e placobutrazol, são capazes de reduzir o comprimento dos entrenós das plantas não afetando quanto ao seu número, podendo causar modificações no tamanho das folhas, tornando-as menores e com um verde mais intenso.

Referente à condutância estomática (gs) nas folhas de pimenta, verificou-se, com a interação 
entre substratos e daminozide, maiores valores em plantas cultivadas na FC, embora em ambos os substratos tenham-se notando frente ao aumento das concentrações, decréscimos nesta variável, atingindo condutância estomática mínima na concentração $7,94 \mathrm{~g} \mathrm{~L}^{-1}$ para a $\mathrm{FC}$ e máxima na concentração 5,11 $\mathrm{g} \mathrm{L}^{-1}$ na areia (Tabela 5), sendo estas reações esperadas, uma vez que também foram verificadas reduções na taxa de transpiração e fotossíntese liquida ao serem tratadas com regulador (Tabela 3). Raros são os trabalhos que avaliam os efeitos destes produtos nas trocas gasosas foliares em ornamentais sendo parâmetros pouco compreendidos e investigados.

Barrett e Nell (1981) relatam que reduções na condutância estomática por reguladores de crescimento podem estar relacionadas com dois mecanismos, sendo estes reduções da transpiração através de uma maior resistência estomática e modificações morfológicas nas folhas provocadas por estes produtos. Orton e Mansfield (1976) relatam que baixas concentrações de daminozide foram capazes de aumentar a concentração interna de $\mathrm{CO}_{2}$ nas folhas de plantas tratadas, sendo capazes de causar inibições na abertura estomática e, em altas concentrações causam danos celulares irreversíveis nestas estruturas provocando reduções na sua eficiência hídrica. De modo geral, o regulador daminozide foi eficaz em reduzir o crescimento e a produção de massa seca das plantas de pimenta ornamental, e em alterar de modo considerado suas características morfofisiológicas.

\section{Conclusões}

Para a obtenção de plantas vigorosas, altura reduzida, caule mais espesso, grande quantidade de frutos e folhas de coloração verde mais intensa, recomenda-se o cultivo de pimenta ornamental cultivar Treasures Red em vasos contendo substrato fibra de côco e pulverizadas por três aplicações com $2 \mathrm{~g} \mathrm{~L}^{-1}$ de daminozide. Concentrações acima desta, provocaram efeitos deletérios na quantidade, comprimento e largura dos frutos, na área foliar e na eficiência fotossintética e condutância estomática chegando a prejudicar o aspecto ornamental $\mathrm{e}$ fisiológico das plantas de pimenta.

\section{Referências}

ALBUQuerque, T. C. S.; MOUCO, M. A. C.; ALBUQUERQUE NETO, A. A. Reguladores de crescimento vegetal na concentração de macronutrientes em videira Itália. Bragantia, Campinas, v. 67, n. 3, p. 553-561, 2008.

ALONI, B.; PASHKAR, T. Antagonistic effects of paclobutrazol and gibberellic acid on growth and some biochemical characteristics of pepper (Capsicum annuum) transplants. Scientia Horticulturae, Amsterdam, v. 33, n. 3-4, p. 167-177, 1987.

ANTUNES, L. E. C.; DUARTE FILHO, J.; BUENO, S. C. S.; MINAMI, K. Tratamento de substratos na produção de mudas de plantas frutíferas. Informe Agropecuário, Lavras, v. 23, n. 216, p. 16-20, 2002.

APPEZZATO, B.; CASTRO, P. R. C. Desenvolvimento e frutificação do tomateiro sob efeito de retardadores de crescimento aplicados em plântulas. Anais da Escola Superior de Agricultura Luiz de Queiroz, Piracicaba, v. 39, n. 2, p. 1020-1037, 1982.

BONACIN, G. A.; RODRIGUES, T. de J. D.; MATTIUZ, C. F. M. Aplicação de retardadores de crescimento em híbridos de girassol ornamental. Revista Brasileira de Horticultura Ornamental, Campinas, v. 12, n. 1, p. $37-$ 42, 2006.

BARRETT, J. E.; NELL, T. A. Transpiration in growth retardant treated poinsettia, bean and tomato. Selected Proceedings of the Florida State Horticultural Society, Florida, v. 94, n. 1, p. 85-87, 1981.

BARRET, J. E. Mecanisms of action. In: TAYAMA, H. K.; LARSON, R. A.; HAMMER, P. A.; ROLLS, T. J. (Ed.). Tips on the use of chemical growth regulators on floriculture crops. Columbus, Ohio Florists' Association, 1992. p. $12-18$

BRUXEL, D.; SILVA, F. C.; LIMA, L. M.; LUZ, J. N. Q.; CARVALHO, J. O. M. Lâminas de irrigação e doses de um condicionador de solo para produção de mudas de tomateiro grupo agroindustrial. Horticultura Brasileira, Brasília, v. 20, n. 2, p. 1317-1318, 2002. Suplemento 2.

CANTLIFFE, D. J. Plug transplant technology. Horticultural Reviews, New York, v. 32, n. 1, p. 397-436, 2008. 
CAVINS, T. J.; GIBSON, J. L.; WHIPKER, B. E.; FONTENO, W. C. pH and EC meters - tool for substrate analysis. North Carolina State University: Florex, 2000. $4 \mathrm{p}$.

CARRIJO, O. A.; LIZ, R. S.; MAKISHIMA, N. Fibra da casca do coco verde como substrato agrícola. Horticultura Brasileira, Brasília, v. 20, n. 4, p. 533-535, 2002.

CUQUEL, F. L.; SABBAGH, M. C.; OLIVEIRA, A. C. B. Control of ornamental sunflower height with daminozide. Semina: Ciências Agrárias, Londrina, v. 31, n. 1, p. 1187-1192, 2010.

DAVIS, T. D.; STEFFENS, G. L.; ANKHLA, N. Triazole plant growth regulators. Horticultural Reviews, Portland, v. 10, n. 3, p. 63-105, 1988.

DAVIS, T. D.; CURRY, E. A. Chemical regulation of vegetative growth. Critical Reviews in Plant Sciences, Philadelphia, v. 10, n. 2, p. 151-188, 1991.

FERREE, D. C.; HALL, F. R. Effects of growth regulators and multiple applications of pesticides on net photosynthesis and transpiration of greenhousegrown apple trees. Journal of the American Society for Horticultural Science, Greensboro, v. 103, n. 1, p. 61-64, 1978.

GROSSI, J. A. S.; MORAES, P. J.; TINOCO, S. A.; BARBOSA, J. G.; FINGER, F. L.; CECON, P. R. Effects of paclobutrazol on growth and fruiting characteristics of cv. Pitanga ornamental Pepper. Acta Horticulturae, Leuven, v. 1, n. 683, p. 333-336, 2005.

GRZESIK, M. Factors influencing the effectiveness of growth regulators in nursery production. Acta Horticulturae, Leuven, v. 2, n. 251, p. 371-376, 1989.

JIANG, H.; FRY, J. Drought responses of perennial Ryegrass treated with plant growth regulators. HortScience, Alexandria, v. 32, n. 2, p. 270-273, 1998.

KÄMPF, A. N. Substrato. In: KÄMPF, A. N. Produção comercial de plantas ornamentais. Guaíba: Agropecuária, 2000. 254 p.

KÄMPF, A. N.; TAKANE, R. J.; SIQUEIRA, P. T. V. Floricultura - Técnicas de preparo de substratos. Brasília: LK Editora e Comunicação, 2006. 132 p.

KOFIDIS, G.; GIANNAKOULA, A.; ILIAS, I. F. Growth, anatomy and chlorophyll fluorescence of coriander plants (Coriandrum sativum L.) treated with prohexadione-calcium and daminozide. Acta Biologica, Cracoviensia, v. 50, n. 2, p. 55-62, 2008.
LOACH, L. Controlling environmental conditions to improved adventitious rooting. In: DAVIS, T. D.; HAISSING, B. E.; SANKLA, N. Adventitious root formation in cuttings. Portland: Dioscorides, 1998. p. 248-273.

MARCUM, K.; JIANG, K. Effects of plant growth regulators on tall fescue rooting and water use. Journal of Turfgrass Management, New York, v. 2, n. 2, p. 13-27, 1997.

MAINARDI, J. C. C. T.; BELLÉ, R. A.; MAINARDI, L. Produção de crisântemo (Dendrathema grandiflora Tzvelev cv. Snowdon) em vaso II: ciclo da cultivar, comprimento, largura e área foliar. Ciência Rural, Santa Maria, v. 34, n. 6, p. 1709-1714, 2004.

NEVES, M. B.; ANDRÉO, Y. S.; WATANABE, A. A.; FAZIO, J. L.; BOARO, C. S. F. Uso de daminozide na produção de girassol ornamental cultivados em vaso. Revista Eletrônica de Agronomia, Garça, v. 16, n. 2, p. 31-37, 2009.

ORTON, P. J.; MANSFIELD, T. A. Studies of the mechanism by which daminozide (B9) inhibits stomatal opening. Journal of Experimental Botany, Oxford, v. 27, n. 1 , p. $125-133,1976$

OZGUR, M. Growth control in cucumber seedlings by growth regulators application. Bulgarian Journal of Agricultural Science, Sofia, v. 17, n. 1, p. 99-106, 2011.

PISARCZYK, J. M.; SPLITTSTOESSER, W. E. Response of tomato to pre-transplanting application of chlormequat, daminozide and ethephon. HostScience, Alexandria, v. 14, n. 1, p. 263-264, 1979.

POBUDKIEWICZ, A.; TREDER, J. Effects of flurprimidol and daminozide on growth and flowering of oriental lily 'Mona Lisa. Scientia Horticulturae, Amsterdam, v. 110, n. 4, p. 328-333, 2006.

RADEMACHER, W. Growth retardants: effects on gibberellin biosynthesis and other metabolic pathways. Annual Review of Plant Physiology and Plant Molecular Biology, Palo Alto, v. 51, n. 1, p. 501-531, 2000.

ROBERTS, B. R; DOMIR, S. C. The influence of daminozide and maleic hydrazide on growth and net photosynthesis of silver maple and american sycamore seedlings. Scientia Horticulturae, Amsterdam, v. 19, n. 3-4, p. 367-372, 1983.

SILVA, E. A.; AMARAL, J. A.; MONTEIRO, R. M. C. Utilização de adubos de liberação lenta na produção de mudas de Helianthus Annus L. cv. Sunbright Supreme. Thesis, São Paulo, ano IX, n. 19, p. 82-91, 2013. Disponível em: <http://www.cantareira.br/thesis2/ed_19/ art_6_girassol.pdf $>$. Acesso em: 28 ago. 2013. 
SILVA, M. P. F.; LOPES, L. C.; MEDINA, P. V. L.; CASALI, V. W. D. Uso do Alar no controle do crescimento de Begonia semperflorens (Link \& Oho). In: CONGRESSO BRASILEIRO DE FLORICULTURA E PLANTAS ORNAMENTAIS, 1., 1980, Campinas. Anais... Campinas: Fundação Cargill, 1980. Disponível em: $<$ http://www.uesb.br/flower/regulador.html $>$. Acesso em: 28 ago. 2013.

STOMMEL, J. R.; BOSLAND, P. Ornamental pepper: Capsicum annuum. In: ANDERSON, N. O. (Ed.). Flower breeding and genetics: issues, challenges and opportunities for the 21st Century. The Netherlands: Springer, Dordrecht, 2006. 822 p.

TINOCO, S. A.; GROSSI, J. A. S.; AZEVEDO, A. A.; BARBOSA, J. G.; SANTOS, N. T. Produção e qualidade de plantas de gerânio zonal (Pelargonium $\mathrm{x}$ hortorum L. H. Bailey) em resposta à aplicação de clormequat, daminozide e paclobutrazol via foliar. Revista Brasileira de Horticultura Ornamental, Campinas, v. 17, n. 2, p. 149-158, 2011.
TOLOTTI, J. C. C.; BELLÉ, R. A.; MAINARDI, L. Produção de crisântemo (Dendranthema grandiflora Tzvelev cv. Snowdon) em vaso I: doses e frequências de aplicação de daminozide. Ciência Rural, Santa Maria, v. 33, n. 6, p. 1045-1051, 2003.

UPNMOOR, I. Cultivo de plantas ornamentais. Guaíba: Agropecuáris, 2003.59 p.

VIEIRA, M. A. Uso de polímeros hidroabsorventes: efeitos sobre a qualidade de substratos hortícolas e crescimento de mudas de pimentão ornamental. 2002. Tese (Doutorado em Agronomia - Produção Vegetal) Faculdade de Agronomia Eliseu Machado. Universidade Federal de Pelotas, Pelotas.

WATANABE, A. A. Desenvolvimento de plantas de girassol (Helianthus annuus l. cv. Pacino) com variação de nutrientes na solução nutritiva e aplicação de Daminozide. 2007. Dissertação (Mestrado em Botânica) - Instituto de Biociências. Universidade Estadual Paulista Júlio de Mesquita Filho, Botucatu. 